\title{
Zum Zusammenhang zwischen der Einstellung der Lehrkraft zu inklusivem Sportunterricht und sozialer Interaktionen von Kindern
}

\author{
Vitus Furrer (D) - Fabian Mumenthaler · Stefan Valkanover • \\ Michael Eckhart $\cdot$ Siegfried Nagel (iD)
}

Eingegangen: 1. November 2020 / Angenommen: 30. April 2021 / Online publiziert: 9. Juli 2021

(C) Der/die Autor(en) 2021

Zusammenfassung Im Zentrum des Beitrags stehen zwei Fragen: 1. Unterscheiden sich Kinder mit und ohne kognitive Beeinträchtigung im inklusiven Sportunterricht in ihren sozialen Interaktionen? 2. Besteht dabei ein Zusammenhang mit der Einstellung der Sportlehrkraft gegenüber inklusiver Bildung? Dazu wurden in einer Querschnittsanalyse 1502 Kinder und 84 Lehrkräfte auf Grundschulstufe befragt. Soziale Netzwerkanalysen zeigen, dass Kinder mit sonderpädagogischem Förderbedarf (SPF) im Sportunterricht signifikant weniger soziale Interaktionen erhalten als Kinder ohne SPF, jedoch gleich viele ausgehende Interaktionen aufweisen. Die sozialen Interaktionen hängen jedoch nicht mit der Einstellung der Lehrkraft zusammen. Auf dieser Basis wird die Rolle der Lehrkraft für die sozialen Interaktionen der Kinder diskutiert.

Schlüsselwörter Inklusive Bildung · Soziale Netzwerke · Einstellungen · Kognitive Beeinträchtigung

Vitus Furrer $(\varangle) \cdot$ Dr. Stefan Valkanover

Fachdidaktikzentrum Sport, PHBern, Bremgartenstrasse 145, 3012 Bern, Schweiz

E-Mail: vitus.furrer@phbern.ch

Dr. Stefan Valkanover

E-Mail: stefan.valkanover@phbern.ch

Fabian Mumenthaler · Prof. Dr. Michael Eckhart

Institut für Heilpädagogik, PHBern, Fabrikstrasse 8, 3012 Bern, Schweiz

Fabian Mumenthaler

E-Mail: fabian.mumenthaler@phbern.ch

Prof. Dr. Michael Eckhart

E-Mail: michael.eckhart@phbern.ch

Prof. Dr. Siegfried Nagel

Institut für Sportwissenschaft, Universität Bern, Bremgartenstrasse 145, 3012 Bern, Schweiz

E-Mail: siegfried.nagel@ispw.unibe.ch 


\title{
On the relationship between teacher attitudes toward inclusive physical education and children's social interactions
}

\begin{abstract}
This paper focuses on two questions: Do children with and without intellectual disabilities in inclusive physical education differ in their social interactions? And does this correlate to the attitude that physical education teachers have toward inclusive education? To this end, in a cross-sectional analysis, 1502 children and 84 teachers at the elementary school level were surveyed. Social network analyses show that children with special educational needs (SEN) receive significantly fewer social interactions in physical education classes than children without SEN but have the same number of outgoing interactions. However, social interactions are not related to teacher attitude. On this basis, the role of the teacher in children's social interactions is discussed.
\end{abstract}

Keywords Inclusive education · Social networks $\cdot$ Attitudes $\cdot$ Intellectual disabilities

\section{Einführung}

Die Policies vieler Länder weisen eine deutliche Tendenz zu mehr inklusiver Bildung auf (Koster et al. 2009), was dem Übereinkommen über die Rechte von Menschen mit Behinderungen, insbesondere des Artikels 24, entspricht. In inklusiven Schulen werden Kinder mit sonderpädagogischem Förderbedarf (SPF) zusammen mit Kindern ohne SPF unterrichtet. Die Förderung der sozialen Partizipation gilt dabei als eines der zentralen Ziele der inklusiven Bildung (Boban und Hinz 2003). In Anlehnung an Koster et al. (2009) wird die soziale Partizipation mit den vier Teilaspekten Freundschaften und Beziehungen, Kontakte und soziale Interaktionen, Akzeptanz durch Mitschülerinnen und -schüler und die Selbstwahrnehmung der Kinder definiert. Das Bedürfnis nach Zugehörigkeit und nach positiver sozialer Partizipation werden von Baumeister und Leary (1995) als grundlegende menschliche Motive dargelegt. Beni et al. (2017) bezeichnen daran anschließend soziale Interaktionen mit Gleichaltrigen als entscheidend für die alltäglichen Schulerfahrungen der Schülerinnen und Schüler. Koster et al. (2009) verstehen unter sozialen Interaktionen bspw. das gemeinsame Spielen, aber auch unerwiderte Interaktionen. Durch soziale Interaktionen erwerben Kinder wichtige soziale Kompetenzen und diese spielen wiederum eine entscheidende Rolle für deren schulisches Wohlbefinden (Garrote und Moser Opitz 2017). Vorliegende Studien zeigen jedoch, dass die soziale Partizipation von Kindern mit SPF in inklusiven Klassen vielfach von Herausforderungen begleitet ist (Garrote et al. 2017). Im Vergleich zu ihren Mitschülerinnen und -schülern haben Kinder mit SPF weniger Interaktionen und Freundschaften und sind weniger akzeptiert. Insbesondere Kinder mit kognitiver Beeinträchtigung sind dabei von sozialer Ausgrenzung bedroht (Schwab 2015). 


\subsection{Soziale Partizipation im inklusiven Sportunterricht}

In Bezug auf die verringerte soziale Partizipation wird dem inklusiven Sportunterricht ein besonderes Potenzial zur Förderung ebendieser zugeschrieben. Unter inklusivem Sportunterricht wird das curriculare, gemeinsame Sporttreiben von Kindern mit und ohne SPF verstanden. Gemeinsamer Unterricht allein ist jedoch noch keine Garantie dafür, dass Kinder mit und ohne SPF in ihrer Klasse gleichberechtigt am sozialen Austausch beteiligt sind (King 2013). Rekaa et al. (2019) berichten in ihrem Review, dass im inklusiven Sportunterricht soziale Ausgrenzung besonders sicht- und erlebbar sein kann. Zudem steht beim Sportunterricht mehr als bei jedem anderen Fach die Körperlichkeit der Lernenden im Mittelpunkt. So kann z. B. Sportlichkeit ein wichtiger Faktor für erfolgreiche soziale Erlebnisse im Sportunterricht sein. Obwohl sich viele Sportlehrkräfte nicht dafür vorbereitet und ausgebildet fühlen (Hutzler et al. 2019), werden in inklusiven Schulen Schülerinnen und Schüler mit SPF aufgenommen. Es gilt deshalb Faktoren zu identifizieren, mit denen die soziale Partizipation zusammenhängt und zu untersuchen, wie diese im Sportunterricht gefördert werden können (De Boer et al. 2012).

\subsection{Zur Bedeutung der Einstellung der Lehrkraft für die soziale Partizipation}

Bezogen auf die unterstützenden Faktoren zeigt die aktuelle Forschung im Kontext der Grundschule, dass die Lehrkraft eine entscheidende Rolle als Moderatorin der sozialen Partizipation von Schülerinnen und Schülern mit SPF spielen kann (Furrer et al. 2020). Dabei wird in der Literatur häufig ein Zusammenhang zwischen einer positiven Einstellung der Lehrkraft und der sozialen Partizipation dieser Zielgruppe postuliert (Schwab 2018; Hutzler et al. 2017). Nach dem Kompetenzmodell von Baumert und Kunter (2013) sind die Einstellungen Teil der professionellen Kompetenzen von Lehrkräften und beinhalten nach dem Dreikomponentenmodell von Ajzen (2005) eine affektive, behaviorale und kognitive Dimension. Laut Baumert und Kunter (2013) wird das unterrichtliche Handeln direkt von den Einstellungen beeinflusst und überträgt sich damit auf die einhergehenden Lernbedingungen - und somit auch auf die sozialen Prozesse - der Schülerinnen und Schüler.

\subsection{Ziel der vorliegenden Studie}

Die Anzahl an Publikationen über Einstellungen zu inklusiver Schulbildung ist in den letzten Jahren stark gestiegen (Lüke und Grosche 2017). Jedoch gibt es nach wie vor relativ wenig Forschungsbemühungen, welche die Rolle der Lehrkraft auf die sozialen Interaktionen der Schülerinnen und Schüler in inklusiven Klassen untersuchen (Garrote et al. 2020; Schwab 2018). Hauptziel der vorliegenden Studie ist, den Zusammenhang über das Ausmaß der sozialen Interaktionen von Schülerinnen und Schülern mit der Einstellung der Sportlehrkraft im inklusiven Sportunterricht auf Grundschulstufe zu untersuchen. Hierzu sollen vorgängig die Ausprägungen der Einstellung der Sportlehrkraft zu inklusivem Sportunterricht dargestellt werden. Des Weiteren ist von Interesse, wie stark die sozialen Interaktionen von Kindern mit SPF im Vergleich zu ihren Mitschülerinnen und -schülern ohne SPF im Sportunterricht 
ausgeprägt sind. Dazu werden netzwerkanalytische Verfahren angewendet. Die Studie konzentriert sich auf die spezifische Subgruppe von Schülerinnen und Schülern mit kognitiver Beeinträchtigung. Soweit uns bekannt ist, gibt es noch keine Studie, die diesen Zusammenhang spezifisch im Sportunterrichtssetting untersucht hat.

\section{Empirische Erkenntnisse zum inklusiven Sportunterricht}

\subsection{Einstellung von Lehrkräften}

Mehrere systematische Review-Artikel befassten sich in den letzten Jahren mit den Einstellungen der Lehrkräfte zu inklusivem Unterricht (z. B. De Boer et al. 2011). Die Studien über die Einstellungen von Lehrkräften variieren je nach Land, Kultur, Bildungssystem und Konzeptualisierung von Inklusion. Demzufolge sind Vergleiche kritisch zu betrachten und es erscheint sinnvoll, die Einstellung von Lehrkräften zu inklusiver Beschulung mit Instrumenten zu messen, die verschiedene Subdimensionen der Lehrkrafteinstellung abdecken, damit ein differenzierteres Bild zu den Einstellungen entsteht (Heyder et al. 2020). Studien bei (Sport-)Lehrkräften zeigen grundsätzlich positive Einstellungen zu inklusiver Bildung (Reuker et al. 2016; Schwab 2018). Reuker et al. (2016) weisen allerdings darauf hin, dass einige Studien im Sportunterricht auch zu ambivalenten Ergebnissen kommen (z. B. Casebolt und Hodge 2010). Dabei wird insbesondere die Einstellung zur Förderung der sozialen Interaktionen zwischen Schülerinnen und Schülern mit und ohne Beeinträchtigung positiv erwähnt, während die konkreten Bedingungen zur Umsetzung eines sozial förderlichen Sportunterrichts durchaus kritisch eingeschätzt werden.

\subsection{Soziale Interaktionen von Kindern mit und ohne SPF}

Studien, welche die sozialen Interaktionserfahrungen im Sportunterricht untersucht haben, sind meist qualitativer Art und beinhalten neben der kognitiven Beeinträchtigung auch Befunde zu anderen Beeinträchtigungen (bspw. körperliche). Goodwin und Watkinson (2000) beschreiben für Kinder mit SPF sowohl ,gute als auch schlechte Tage“ im inklusiven Sportunterricht. Ähnlich berichten auch Goodwin (2001) und Butler und Hodge (2004) von sowohl unterstützenden als auch exkludierenden Erfahrungen für Kinder mit SPF. In einer quantitativen Studie mit 20 inklusiven Schulklassen mit mindestens einem Kind mit kognitiver Beeinträchtigung (1.-3. Grundschuljahr) kommt Garrote (2016) zum Ergebnis, dass Kinder mit kognitiver Beeinträchtigung signifikant weniger Interaktionen erhalten als ihre Peers ohne kognitive Beeinträchtigung - obwohl sich die Werte hinsichtlich der ausgehenden Interaktionen nicht unterscheiden. Das heißt, dass Kinder mit kognitiver Beeinträchtigung zwar gleich oft die Interaktion mit Mitschülerinnen und -schülern suchen, sich dabei aber signifikant weniger Interaktionen ergeben.

Zusammenfassend lässt sich festhalten, dass Kinder mit SPF in Schulklassen weniger Interaktionen erhalten als Kinder ohne SPF. Dieser Befund könnte als Etikettierungseffekt interpretiert werden (Schwab 2019). Entscheidend für eine solche Interpretation ist jedoch, dass möglichst alle wichtigen Faktoren in der Analyse 
berücksichtigt werden. Wichtige netzwerkinhärente Strukturen, wie z. B. die Homophilie - als Tendenz eines Menschen, sich stärker zu Mitmenschen hingezogen zu fühlen, die ihm ähnlich sind (McPherson et al. 2001) -, wurden jedoch bislang kaum berücksichtigt (Ausnahmen aus dem Klassenzimmerunterricht sind Henke et al. 2017a und Mamas et al. 2020). Der Fokus auf netzwerkinhärente Strukturen kann aufzeigen, dass Interaktionen vermehrt zwischen Menschen stattfinden, die das gleiche Geschlecht haben oder sich in ihrem Alter oder Leistungsniveau ähneln (Zander und Hannover 2014). In inklusiven Klassen ist es folglich für Schülerinnen und Schüler mit SPF schwieriger, Gleichaltrige zu finden, die ähnliche individuelle Merkmale und Leistungsniveaus aufweisen (Frostad und Pijl 2007). Es ist davon auszugehen, dass der soziale Mechanismus, der zur häufig konstatierten Exklusion von Schülerinnen und Schülern mit SPF führt, nicht ein Etikettierungseffekt ist, sondern das Resultat von Homophilie sein könnte. In sozialen Netzwerkanalysen können diese inhärenten Strukturen kontrolliert werden.

\subsection{Der Zusammenhang zwischen sozialen Interaktionen und der Einstellung der Lehrkraft}

Obwohl eine positive Einstellung der Lehrkräfte zu inklusiver Bildung oft als Voraussetzung für erfolgreiche soziale Interaktionen angesehen (Schwab 2018) und theoretisch durch das Kompetenzmodell von Baumert und Kunter (2013) gestützt wird, gibt es kaum empirische Belege für diese Annahme (Schwab 2018; Heyder et al. 2020). Im Bereich der Grundschulbildung liegen nur vereinzelte quantitative (Heyder et al. 2020; Monsen et al. 2014), qualitative (Ben-Yehuda et al. 2010) und Mixed Methods Studien (Garrote et al. 2020) vor, die den Zusammenhang zwischen der Einstellung von Lehrkräften zur inklusiven Bildung und den sozial-emotionalen Schulerfahrungen von Schülerinnen und Schülern analysieren. Aufgrund der dünnen empirischen Befundlage zu den sozialen Interaktionen werden im Folgenden zusätzliche Aspekte der sozialen Partizipation nach Koster et al. (2009) miteinbezogen.

Dabei kann festgestellt werden, dass Schülerinnen und Schüler, die von Lehrkräften mit einer sehr positiven Einstellung zu inklusiver Bildung unterrichtet werden, über eine höhere Zufriedenheit im Klassenzimmer berichten als Schülerinnen und Schüler von Lehrkräften mit einer weniger positiven Einstellung (Monsen et al. 2014). Außerdem finden Heyder et al. (2020) in ihrer Studie heraus, dass nur einzelne Dimensionen der Einstellung (vgl. Abschn. 1.2) mit dem sozial-emotionalen Wohlbefinden der Schülerinnen und Schüler im inklusiven Unterricht zusammenhängen. Einzig die explizite Einstellung zum Nutzen von inklusiver Bildung gegenüber Sonderbeschulung hängt mit einer geringeren Differenz in der sozialen Partizipation von Kindern mit und ohne SPF zusammen. Im Gegensatz dazu finden Garrote et al. (2020) in einer Längsschnittstudie keinen Effekt der Lehrkrafteinstellung auf die soziale Akzeptanz im Klassenzimmer.

Unklar bleibt, ob ein allfälliger Zusammenhang zwischen dem Einstellungsniveau der Lehrkräfte auf Kontext- bzw. Klassenebene und dem SPF der Schülerinnen und Schüler auf der Individualebene vorliegt. Unseres Wissens gibt es nur vereinzelte Studien, die solche Cross-Level-Interaktionen untersuchten. So haben Lehrkräfte von Klassen, in welchen sich die soziometrischen Werte von Kindern mit und ohne 
SPF nicht unterscheiden, eine positivere Einstellung zu inklusiver Bildung (BenYehuda et al. 2010). Heyder et al. (2020) finden bei der Subdimension zum Nutzen von inklusiver Bildung gegenüber Sonderbeschulung ebenfalls eine entsprechende Cross-Level-Interaktion. Studien im Setting des inklusiven Sportunterrichts wurden keine gefunden.

\section{Fragestellungen}

Obwohl im Bereich der sozialen Interaktionen von Schülerinnen und Schülern mit SPF im inklusiven Unterricht schon viel Wissen vorhanden ist, gibt es nach wie vor Forschungslücken in Bezug auf den inklusiven Sportunterricht mit Kindern mit kognitiver Beeinträchtigung. Ziel des vorliegenden Artikels ist es, den Zusammenhang zwischen der Einstellung der Lehrkraft und den sozialen Interaktionen von Kindern (mit SPF) im inklusiven Sportunterricht auf Grundschulstufe in der Schweiz $\mathrm{zu}$ untersuchen.

Es ergeben sich drei forschungsleitende Fragestellungen:

1. Wie ist die Einstellung von Lehrkräften zu inklusivem Sportunterricht einzustufen?

2. Wie sind die sozialen Interaktionen (eingehende und ausgehende) von Schülerinnen und Schülern mit und ohne SPF im inklusiven Sportunterricht ausgeprägt?

3. Inwieweit steht die Einstellung von Lehrkräften zu inklusivem Sportunterricht in einem Zusammenhang mit den sozialen Interaktionen (eingehende und ausgehende) der Schülerinnen und Schüler mit und ohne SPF?

\section{Methode}

Die vorliegenden Analysen sind in das Projekt Soziale Partizipation im Sport (SoPariS) des Schweizerischen Nationalfonds eingebettet (2018-2021). Das Projekt befasst sich mit der sozialen Partizipation von Schülerinnen und Schülern mit kognitiver Beeinträchtigung ${ }^{1}$ im inklusiven Sportunterricht in Deutschschweizer Grundschulklassen (3. bis 6. Klasse). Im Jahr 2019 wurde eine Querschnittsstudie mit quantitativen Fragebogen für Schülerinnen, Schüler und Sportlehrkräfte durchgeführt. Die große Heterogenität der Gruppe der Schülerinnen und Schüler mit SPF wurde verringert, indem die Stichprobe auf Schülerinnen und Schüler mit kognitiver Beeinträchtigung beschränkt wurde, wobei die Bandbreite der Fähigkeiten der Schülerinnen und Schüler innerhalb der Gruppe der Kinder mit kognitiver Beeinträchtigung immer noch sehr groß ist.

\footnotetext{
1 Kinder mit kognitiver Beeinträchtigung verfügen in der Schweiz über einen entsprechenden Sonderschulstatus. D.h., dass sie aufgrund einer Abklärung (i.d. R. mit dem Standardisierten Abklärungsverfahren) ausgewiesenen Anspruch auf verstärkte Maßnahmen haben.
} 
Tab. 1 Deskriptive Statistik der Schülerinnen und Schüler und Lehrkraft Stichproben ( $N=84$ Klassen, 3. bis 6. Grundschulklasse)

\begin{tabular}{llll}
\hline & $n$ & Alter (SD) & Weiblich $(\%)$ \\
\hline Kinder ohne SPF & 1399 & $11,18(1,08)$ & 52,0 \\
Kinder mit SPF & 103 & $11,76(1,13)$ & 37,9 \\
Lehrkräfte & 84 & $37,73(11,7)$ & 67,9 \\
\hline
\end{tabular}

SPF Sonderpädagogischer Förderbedarf

\subsection{Stichprobe}

An der Erhebung nahmen insgesamt 109 inklusive Klassen aus der Schweiz teil. Total wurden 1961 Schülerinnen und Schüler und 107 Lehrkräfte befragt. Aufgrund unvollständiger Fragebogen von Schülerinnen, Schülern und Lehrkräften waren systematische Datenverluste zu verzeichnen. Des Weiteren kam es wegen nicht konvergierender Exponential Random Graph Models (ERGMs) zum Ausschluss von elf Klassen (vgl. Abschn. 4.4). Dies reduzierte die Stichprobe auf 1502 Kinder und 84 Sportlehrkräfte in insgesamt 84 Schulklassen (vgl. Tab. 1). Die Klassengröße betrug zwischen 10 und 25 Schülerinnen und Schüler $(M=17,89, S D=3,5)$. In jeder Klasse hatte es zwischen einer resp. einem und vier Schülerinnen und Schüler mit kognitiver Beeinträchtigung.

\subsection{Datenerhebung}

Grundschulklassen aus 13 verschiedenen Kantonen nahmen an dieser Studie teil. Die Schulleitung und Lehrkräfte sowie die Eltern gaben ihre Zustimmung zur Studie. Jedes Item aus dem Fragebogen wurde im Klassenzimmer laut von der Projektleitung vorgelesen und der Fragebogen wurde gemeinsam mit allen Kindern durchgearbeitet. Klassenlehrkräfte, schulische Heilpädagoginnen und Heilpädagogen und speziell geschulte Master-Studierende unterstützten die Schülerinnen und Schüler während des Ausfüllens. Die Angaben der Lehrkräfte wurden mittels Online-Fragebogen erhoben. Die Studie wurde von der Ethikkommission der Fakultät der Universität Bern genehmigt.

\subsection{Messinstrumente}

\subsubsection{Soziale Interaktionen im Sportunterricht}

In der vorliegenden Studie wurde der soziometrische Bewertungsfragebogen Sozio verwendet (Eckhart 2012). Die Schülerinnen und Schüler wurden gebeten, auf einer 5 -stufigen Likert-Skala $(0=$ fast nie, $4=$ sehr viel $)$ anzugeben, wie häufig sie im Sportunterricht mit jeder Mitschülerin und jedem Mitschüler sprechen bzw. spielen. Obwohl Interaktionen grundsätzlich gegenseitig stattfinden, wird aufgrund des gerichteten Messinstruments von ein- und ausgehenden Interaktionen gesprochen, da es um wahrgenommene Interaktionen aus individueller Sicht geht.

Für die Berechnung der Popularität (resp. Aktivität) eines Kindes wurde die Summe aller erhaltenen (resp. ausgehenden) Sprechkontakte durch die Anzahl der 
möglichen Sprechkontakte geteilt. Für die Berechnungen der sozialen Netzwerke mittels ERGMs wurden die Rohwerte für die Interaktionen nach gängiger Praxis für Netzwerke dichotomisiert (vgl. Borgatti und Quintane 2018). Die Werte für fast nie, selten und manchmal wurden als 0 , viel und sehr viel als 1 codiert.

\subsubsection{Einstellung der Lehrkraft zu inklusivem Sportunterricht}

Zur Erfassung der Einstellung zu inklusivem Sportunterricht wurde der Einstellungsfragebogen zu Inklusion für Lehrkräfte (EFI-L; Seifried und Heyl 2016) verwendet. Die Originalfragen von Seifried und Heyl (2016) wurden in einer Vorstudie um den Kontext Sportunterricht ergänzt. Die dreifaktorielle Struktur umfasst die beiden schülerbezogenen Dimensionen Fachliche Förderung im inklusiven Unterricht (Beispielitem: Kinder mit kognitiven Beeinträchtigungen würden im integrativen Sportunterricht letztendlich besser gefördert) und Soziale Inklusion im Unterricht (Beispielitem: Kinder mit kognitiven Beeinträchtigungen würden sich im integrativen Sportunterricht allein und ausgeschlossen fühlen [invers kodiert]) sowie die lehrkraftbezogene Dimension Persönliche Bereitschaft zu inklusivem Unterricht (Beispielitem: Ich kann mir vorstellen, im kommenden Schuljahr wieder integrativen Sportunterricht zu unterrichten). Jede Skala umfasste zwischen vier und sechs Items (insgesamt 15 Items). Die Lehrkräfte mussten auf einer 6-stufigen Likert-Skala angeben, wie fest sie einer Aussage zustimmten $(0=$ stimme ganz und gar nicht $z u$ bis $5=$ stimme voll und ganz zu). Analysen der internen Konsistenz ergaben ein Cronbach's Alpha zwischen 0,76 und 0,83 für die drei Subdimensionen, wobei sie signifikant korrelieren $(r=0,22$ bis $0,55, p \leq 0,05, N=84)$.

\subsubsection{Psychomotorische Ungeschicklichkeit}

Die psychomotorische Ungeschicklichkeit soll in den folgenden Analysen als Kontrollvariable eingesetzt werden. Die Fragen für die qualitative Beurteilung des Bewegungsverhaltens basieren auf der von Valkanover (2005) adaptierten Checkliste motorischer Verhaltensweisen von Schilling (1976). Für das vorliegende Projekt wurde die verwendete Skala Psychomotorische Ungeschicklichkeit auf drei Items adaptiert. Sie beschreibt unbeholfenes und ungeschicktes Bewegungsverhalten von Schülerinnen und Schülern. Die drei Items (Beispielitem: Das Bewegungsverhalten des Kindes bei selbstgewählten (Bewegungs-)Aufgaben ist ungeschickt) waren von den Lehrkräften auf einer 5-stufigen Likert-Skala von 0 (trifft überhaupt nicht zu) bis 4 (trifft voll und ganz zu) zu bewerten. Analysen der internen Konsistenz ergaben ein Cronbach's Alpha von 0,87.

\subsection{Datenanalyse}

Die Beantwortung der ersten Fragestellung erfolgt durch den Vergleich des theoretischen Skalenmittelwerts mit dem empirischen Mittelwert der Stichprobe (vgl. Schwab 2015) und wird separat für den Gesamtscore und die drei Subdimensionen der Einstellungsmessung nach Seifried und Heyl (2016) analysiert. Dabei werden 
Tab. 2 Übersicht über die Modellparameter

\begin{tabular}{|c|c|c|c|}
\hline & $\begin{array}{l}\text { Modell- } \\
\text { parameter }^{\mathrm{a}}\end{array}$ & Beschreibung & Visualisierung $^{\mathrm{b}}$ \\
\hline \multirow{6}{*}{$\begin{array}{l}\text { Strukturelle, } \\
\text { netzwerk- } \\
\text { endogene } \\
\text { Faktoren }\end{array}$} & Dichte & $\begin{array}{l}\text { Der Koeffizient gibt die Log Odds aus, für die Ten- } \\
\text { denz, überhaupt einen Kontakt zu senden. }\end{array}$ & \\
\hline & Reziprozität & $\begin{array}{l}\text { Die Wahrscheinlichkeit, dass Person } i \text { an Person } j \\
\text { einen Kontakt sendet, steigt, gegeben dass } j \text { einen } \\
\text { Kontakt an } i \text { sendet. }\end{array}$ & \\
\hline & Popularität & $\begin{array}{l}\text { Maß für eingehende Interaktionen. Gezählt wird die } \\
\text { Struktur, bei der zwei verschiedene Kontakte erhalten } \\
\text { werden. Ein hoher Wert sagt aus, dass es im Netzwerk } \\
\text { Akteure gibt, die diese Struktur überzufällig häufig } \\
\text { aufweisen. }\end{array}$ & \\
\hline & Aktivität & $\begin{array}{l}\text { Maß für ausgehende Interaktionen. Gezählt wird die } \\
\text { Struktur, bei der zwei verschiedene Kontakte gesendet } \\
\text { werden. Ein hoher Wert sagt aus, dass es im Netzwerk } \\
\text { Akteure gibt, die diese Struktur überzufällig häufig } \\
\text { aufweisen. }\end{array}$ & \\
\hline & Transitivität & $\begin{array}{l}\text { Die Wahrscheinlichkeit, dass } i \text { einen Kontakt an } k \\
\text { sendet, steigt, wenn bereits die Verbindung } i \rightarrow j \rightarrow k \\
\text { besteht. }\end{array}$ & \\
\hline & Zwei-Pfad & $\begin{array}{l}\text { Eine Struktur, die in der Transitivität enthalten ist, } \\
\text { ist der Zwei-Pfad, also die Verbindung } i \rightarrow j \rightarrow k \text {, die } \\
\text { bestehen muss, damit daraus eine transitive Triade } \\
\text { entstehen kann. Indem für Zwei-Pfad kontrolliert wird, } \\
\text { wird sichergestellt, dass bei Transitivität wirklich auch } \\
\text { nur der Effekt von Transitivität gemessen wird. }\end{array}$ & \\
\hline \multirow[t]{6}{*}{$\begin{array}{l}\text { Exogene } \\
\text { Faktoren }\end{array}$} & $\begin{array}{l}\text { Geschlechter- } \\
\text { homophilie }\end{array}$ & $\begin{array}{l}\text { Ein Kontakt zwischen zwei Akteuren wird wahrschein- } \\
\text { licher, wenn diese das gleiche Geschlecht haben. }\end{array}$ & \\
\hline & $\begin{array}{l}\text { Alter, ab- } \\
\text { solute } \\
\text { Differenz }\end{array}$ & $\begin{array}{l}\text { Ein Kontakt zwischen zwei Akteuren wird wahrschein- } \\
\text { licher, wenn diese ein ähnliches Alter haben (Alterssi- } \\
\text { milarität). }\end{array}$ & \\
\hline & $\begin{array}{l}\text { Motorisch } \\
\text { ungeschickt }\end{array}$ & $\begin{array}{l}\text { Eine hohe Ausprägung im Attribut ungeschickt verrin- } \\
\text { gert die Wahrscheinlichkeit, einen Kontakt zu senden } \\
\text { oder zu erhalten. }\end{array}$ & \\
\hline & $\begin{array}{l}\text { Motorisch } \\
\text { ungeschickt } \\
\text { (Tail-to-Head } \\
\text { Differenz) }\end{array}$ & $\begin{array}{l}\text { Misst die Differenz im Attribut ungeschickt, wobei } \\
\text { der Wert vom receiver vom Wert des senders abge- } \\
\text { zogen wird. Ein positiver Koeffizient sagt aus, dass } \\
\text { ungeschicktere eher Kontakte zu geschickteren senden. }\end{array}$ & \\
\hline & $\begin{array}{l}\text { SPF (einge- } \\
\text { hend) }\end{array}$ & $\begin{array}{l}\text { Misst den Effekt von SPF auf die Wahrscheinlichkeit, } \\
\text { einen Kontakt zu erhalten im Vergleich mit Kindern } \\
\text { ohne SPF. }\end{array}$ & \\
\hline & $\begin{array}{l}\text { SPF (aus- } \\
\text { gehend) }\end{array}$ & $\begin{array}{l}\text { Misst den Effekt von SPF auf die Wahrscheinlichkeit, } \\
\text { einen Kontakt zu senden im Vergleich mit Kindern } \\
\text { ohne SPF. }\end{array}$ & \\
\hline
\end{tabular}

$S P F$ Sonderpädagogischer Förderbedarf

${ }^{a}$ Die Modellparameter werden auch als Effekte bezeichnet (synonyme Verwendung)

${ }^{\mathrm{b}}$ Die Visualisierungen mit Buchstaben kennzeichnen endogene Strukturen, jene mit schwarz/weiß/grau exogene Strukturen. Schwarz: Individuum besitzt dieses Attribut (kategorial); grau: Ausprägung des Attributs (kardinal); weiß: Ausprägung des Attributs spielt keine Rolle. Die endogenen Faktoren werden von Robins und Lusher (2013) für Netzwerkanalysen vorgeschlagen, die exogenen Faktoren sind interessengeleitet 
Einstichproben $t$-Tests anhand des theoretischen Mittelwerts von 2,5 mit SPSS gerechnet (IBM Corp 2020).

Die zweite Fragestellung bezüglich sozialer Interaktionen wird mittels ERGMs beantwortet (R-package ergm, Hunter et al. 2008). ERGMs sind statistische Modelle, welche auf der Idee von logistischen Regressionen aufbauen, jedoch die endogenen und exogenen Abhängigkeiten von sozialen Netzwerken berücksichtigen (Lusher et al. 2013). Die endogene Abhängigkeit bezieht sich auf die Struktur innerhalb eines Netzwerkes (bspw. Reziprozität). Als exogene Variablen werden Personenattribute betrachtet (bspw. Homophilie). Das Ziel dieser Analyse ist es, einen möglichst genauen Schätzer für den Einfluss des SPF auf die sozialen Interaktionen zu erhalten, indem in jedem Netzwerk die übrigen endogenen und exogenen Abhängigkeiten kontrolliert werden. Robins und Lusher (2013) schlagen vor, für die in Tab. 2 aufgeführten endogenen Parameter zu kontrollieren.

Nach Robins und Lusher (2013) soll die Modellspezifikation theoriegeleitet erfolgen. Für jede Klasse wurde zuerst das Vollmodell mit allen Modellparametern aus Tab. 2 gerechnet. Wenn das Modell nicht konvergierte, wurden die Effekte Popularität, Aktivität und Zwei-Pfad herausgenommen und erneut gerechnet, da diese Parameter für die vorliegenden Interaktionsnetzwerke als weniger bedeutsam eingestuft werden als Reziprozität und Transitivität. Wenn dieses Modell konvergierte, wurden schrittweise die herausgenommenen Effekte wieder eingefügt und nach dem besten Modell-Fit gesucht (,backward-forward“"Modellselektion nach Snijders et al. 2010). Als Entscheidungshilfe für die Modellauswahl diente der AIC und die Goodness-of-Fit-Statistiken. Eine Klasse wurde aufgrund eines ungenügenden ModellFits von der Analyse ausgeschlossen. Die Effekte für eingehende und ausgehende Interaktionen von Kindern mit SPF konnten nicht in jedem Modell geschätzt werden, da sie z. T. hoch mit anderen Variablen korrelierten oder den tiefst möglichen Wert hatten (d.h. keine eingehenden Interaktionen), wodurch ihr Effekt bei -infinity festgesetzt wurde.

In dieser Analyse werden die einzelnen Klassen als Fallstudien betrachtet. Um die klassenübergreifenden Prozesse in den sozialen Netzwerken aus der vorliegenden Studie zusammenzufassen, werden deshalb für die zweite Fragestellung MetaAnalysen gerechnet (R-package metafor, Viechtbauer 2010). Wie von An (2015) vorgeschlagen, werden Fixed und Random Effects Modelle der Meta-Analyse präsentiert.

Um den Zusammenhang der Einstellung der Sportlehrkraft mit den sozialen Interaktionen der Kinder zu untersuchen, werden in der dritten Fragestellung in einem ersten Schritt Multilevel-Regressionen gerechnet, um den absoluten Effekt der Lehrkrafteinstellung auf die eingehenden und ausgehenden Interaktionen der Kinder zu schätzen (R-package nlme, Pinheiro et al. 2020). Dabei werden die Rohwerte der Popularität und Aktivität berücksichtigt, um keinen Informationsverlust durch die Dichotomisierung zu erhalten (vgl. Abschn. 4.3.1). Die Cross-Level-Interaktionen der Einstellungen mit dem SPF werden in einem zweiten Schritt mittels Mixed Effects Modellen gerechnet, bei denen die Einstellungswerte der Lehrkräfte als Moderatorvariable auf den Parameter SPF (eingehend) und SPF (ausgehend) aus den ERGM Berechnungen aus Fragestellung 2 regressiert werden. Hierfür muss beachtet werden, dass die abhängige Variable ein Koeffizient ist, der im Vergleich zu 
einer Baseline (= Kinder ohne SPF) zu sehen ist. D.h., Kinder mit SPF erhalten weniger oder mehr Interaktionen im Vergleich zu den Kindern ohne SPF, in Abhängigkeit von der Einstellung der Lehrkraft. Beim Mixed Effects Modell wird deshalb eine Cross-Level-Interaktion geschätzt, die gegenüber herkömmlichen MultilevelRegressionen zusätzlich die endogenen und exogenen Abhängigkeiten in den Netzwerken kontrolliert.

\section{Resultate}

\subsection{Einstellung der Sportlehrkräfte}

Für die erste Fragestellung wurden die Einstellungswerte der Lehrkräfte zu inklusivem Sportunterricht mit Kindern mit kognitiver Beeinträchtigung untersucht. In Tab. 3 sind die Mittelwerte, Standardabweichungen und die Spannweite des Gesamtscores sowie der Subdimensionen des EFI-L Einstellungsfragebogens ersichtlich. Die Analysen der Einstichproben $t$-Tests zeigen, dass sich die lehrkraftbezogene Dimension Persönliche Bereitschaft zu inklusivem Unterricht $(t(83)=14,69$, $p<0,001, d=1,60$ ), die schülerbezogene Dimension Soziale Inklusion im Unterricht $(t(83)=12,64, p<0,001, d=1,38)$ und die Dimension Fachliche Förderung im inklusiven Unterricht $(t(83)=4,85, p<0,001, d=0,53)$ signifikant vom theoretischen Mittelwert unterscheiden. Es handelt sich um mittlere bis große Effekte (Cohen 1988). Alle Subdimensionen der Einstellung von Lehrkräften sind signifikant höher als der theoretische Mittelwert von 2,5.

\subsection{Soziale Interaktionen im Sportunterricht}

Alle strukturellen Parameter der zweiten Fragestellung sind hochsignifikant, was dafürspricht, dass man diese endogenen Strukturen kontrolliert (vgl. Tab. 4). Die sozialen Netzwerke im Sportunterricht aus der vorliegenden Studie sind dadurch geprägt, dass signifikant weniger als die Hälfte aller möglichen Interaktionen genannt werden (Dichte). Des Weiteren tendieren die Kinder dazu, erhaltene Interaktionen zu erwidern (Reziprozität) und mögliche Triaden zu schließen (Transitivität). Die Netzwerke zeichnen sich zudem dadurch aus, dass es besonders populäre und aktive Kinder gibt. Auch die exogenen Variablen beeinflussen die Netzwerke signifikant: Erwartungsgemäß ist der Parameter Geschlechterhomophilie signifikant positiv, was bedeutet, dass Mädchen v.a. mit Mädchen und Jungen v.a. mit Jungen interagie-

Tab. 3 Deskriptive Statistik zu den Einstellungen der Sportlehrkräfte (theoretisches Minimum=0, theoretisches Maximum=5)

\begin{tabular}{llllll}
\hline EFI-L & $N$ & Mittelwert & Std.-Abweichung & Minimum & Maximum \\
\hline Fachliche Förderung & 84 & 3,00 & 0,95 & 0,67 & 4,67 \\
Soziale Inklusion & 84 & 3,76 & 0,92 & 1,00 & 5,00 \\
Persönliche Bereitschaft & 84 & 3,82 & 0,83 & 0,80 & 5,00 \\
Gesamtscore & 84 & 3,53 & 0,71 & 1,63 & 4,70 \\
\hline
\end{tabular}

EFI-L Einstellungsfragebogen zu Inklusion für Lehrkräfte (Seifried und Heyl 2016) 
Tab. 4 Fixed und Random Effects Meta-Analysen der ERGMs

\begin{tabular}{|c|c|c|c|c|c|c|}
\hline & Effekt & $\begin{array}{l}\text { Anzahl } \\
\text { Netzwerke }\end{array}$ & Fixed Effects & OR & Random Effects & OR \\
\hline \multirow{6}{*}{$\begin{array}{l}\text { Strukturelle, } \\
\text { netzwerk- } \\
\text { endogene } \\
\text { Faktoren }\end{array}$} & Dichte & 84 & $-3,12(0,10) * * *$ & - & $-2,98(0,15) * * *$ & - \\
\hline & Reziprozität & 84 & $1,64(0,06) * * *$ & - & $1,64(0,08) * * *$ & - \\
\hline & Popularität & 73 & $0,12(0,01) * * *$ & - & $0,08(0,02) * * *$ & - \\
\hline & Aktivität & 63 & $0,27(0,01) * * *$ & - & $0,27(0,01) * * *$ & - \\
\hline & Transitivität & 84 & $0,68(0,04) * * *$ & - & $0,68(0,05) * * *$ & - \\
\hline & Zwei-Pfad & 73 & $-0,15(0,01) * * *$ & - & $-0,16(0,01) * * *$ & - \\
\hline \multirow[t]{6}{*}{$\begin{array}{l}\text { Exogene } \\
\text { Faktoren }\end{array}$} & $\begin{array}{l}\text { Geschlechter- } \\
\text { homophilie }\end{array}$ & 84 & $1,49(0,04) * * *$ & 4,44 & $1,83(0,09) * * *$ & 6,23 \\
\hline & $\begin{array}{l}\text { Alter, absolute } \\
\text { Differenz }\end{array}$ & 84 & $-0,18(0,03) * * *$ & 0,84 & $-0,20(0,04) * * *$ & 0,82 \\
\hline & $\begin{array}{l}\text { Motorisch unge- } \\
\text { schickt }\end{array}$ & 84 & $-0,18(0,01) * * *$ & 0,84 & $-0,22(0,02) * * *$ & 0,80 \\
\hline & $\begin{array}{l}\text { Motorisch unge- } \\
\text { schickt (Tail-to- } \\
\text { Head Differenz) }\end{array}$ & 84 & $0,09(0,01) * * *$ & 1,09 & $0,11(0,02) * * *$ & 1,12 \\
\hline & SPF (eingehend) & 73 & $-0,25(0,09) * *$ & 0,78 & $-0,29(0,11) *$ & 0,75 \\
\hline & SPF (ausgehend) & 69 & $0,13(0,06) *$ & 1,14 & $0,08(0,11)$ & 1,08 \\
\hline
\end{tabular}

$S P F$ Sonderpädagogischer Förderbedarf, OR Odds Ratio

In Klammern sind die Standardfehler

$* * * p \leq 0,001, * * p \leq 0,01, * p \leq 0,05$

ren. Der negative Koeffizient beim Alter bedeutet, dass geringere Altersunterschiede Interaktionen im Sportunterricht wahrscheinlicher machen. Der negative Koeffizient beim Parameter zur motorischen Ungeschicklichkeit zeigt, dass ungeschicktere Kinder im Sportunterricht weniger Interaktionen haben. Der zweite Koeffizient zu motorischer Ungeschicklichkeit (Tail-to-Head Differenz) sagt aus, dass ungeschicktere Kinder bei den sozialen Interaktionen häufiger geschicktere Kinder wählen als umgekehrt.

Wenn für alle aufgeführten Parameter kontrolliert wird, erhalten Kinder mit SPF immer noch signifikant weniger Interaktionen im Sportunterricht als Kinder ohne SPF. Die beiden gerechneten Modelle Fixed und Random Effects unterscheiden sich kaum, was darauf schließen lässt, dass die Modelle gut zu den Daten passen. Bei den ausgehenden Interaktionen nennen Kinder mit SPF leicht mehr Interaktionen als Kinder ohne SPF, im Fixed Effects Modell ist dieser Effekt auf dem 5\%-Niveau signifikant. Die Koeffizienten bei den exogenen Variablen können gleich wie bei logistischen Regressionsmodellen interpretiert werden: Kinder mit SPF erhalten um $22 \%$ resp. $25 \%$ weniger wahrscheinlich eine Interaktion (Fixed Effects: $\exp [-0,25]=0,78$; Random Effects: $\exp [-0,29]=0,75)$, als Kinder ohne SPF. Bei den ausgehenden Interaktionen ist es um 14\% resp. $8 \%$ wahrscheinlicher, dass Kinder mit SPF eine Interaktion senden als Kinder ohne SPF (Fixed Effects: exp $[0,13]=1,14$; Random Effects: $\exp [0,08]=1,08)$. 


\subsection{Zusammenhang der Einstellung der Sportlehrkraft mit den sozialen Interaktionen}

Für die dritte Fragestellung wurden zuerst Multilevel-Regressionen mit den Rohwerten der eingehenden (Popularität) und ausgehenden Interaktionen (Aktivität) gerechnet (vgl. Tab. 5). Die Subdimension Persönliche Bereitschaft zu inklusivem Unterricht ist die einzige Variable mit einem positiven Vorzeichen. Es gibt jedoch keine signifikanten Zusammenhänge zwischen der Einstellung der Lehrkraft und dem Level an sozialen Interaktionen der Kinder auf Klassenebene. Der $p$-Wert für den Gesamtscore liegt bei 0,09 für Popularität und 0,08 für Aktivität und bei der Subdimension Fachliche Förderung im inklusiven Unterricht bei 0,14 resp. 0,12. Die übrigen $p$-Werte der Subdimensionen sind zwischen 0,73 und 0,97. Die Level 1 Parameter wurden hier nur als Kontrollvariablen einbezogen. Die für die vorliegende Untersuchung relevanten Ergebnisse zu diesen Faktoren wurden jedoch bereits unter 5.2. erläutert, wo netzwerktypische Abhängigkeiten berücksichtigt werden konnten.

Tab. 5 Multilevel-Regressionen der eingehenden Interaktionen (Popularität; Modell 1 und 2) und ausgehenden Interaktionen (Aktivität; Modell 3 und 4) der Schülerinnen und Schüler (unstandardisierte Koeffizienten)

\begin{tabular}{|c|c|c|c|c|}
\hline & $\begin{array}{l}\text { Modell } 1 \text { Popula- } \\
\text { rität }\end{array}$ & $\begin{array}{l}\text { Modell } 2 \text { Popula- } \\
\text { rität }\end{array}$ & $\begin{array}{l}\text { Modell } 3 \text { Aktivi- } \\
\text { tät }\end{array}$ & $\begin{array}{l}\text { Modell } 4 \text { Aktivi- } \\
\text { tät }\end{array}$ \\
\hline \multicolumn{5}{|l|}{ Level 1} \\
\hline Mit SPF & $-0,11(0,01) * * *$ & $-0,11(0,01) * * *$ & $-0,01(0,02)$ & $-0,01(0,02)$ \\
\hline Mädchen & $-0,02(0,01) * * *$ & $-0,02(0,01) * * *$ & $0,02(0,01) *$ & $0,02(0,01) *$ \\
\hline $\begin{array}{l}\text { Motorisch unge- } \\
\text { schickt }\end{array}$ & $-0,04(0,00) * * *$ & $-0,04(0,00) * * *$ & $-0,03(0,00) * * *$ & $-0,03(0,00) * * *$ \\
\hline Alter & $0,00(0,00)$ & $0,00(0,00)$ & $0,02(0,01) * *$ & $0,02(0,01) * *$ \\
\hline \multicolumn{5}{|l|}{ Level 2} \\
\hline $\begin{array}{l}\text { EFI-L Gesamt- } \\
\text { score }\end{array}$ & $-0,02(0,01)$ & - & $-0,02(0,01)$ & - \\
\hline $\begin{array}{l}\text { EFI-L Fachliche } \\
\text { Förderung }\end{array}$ & - & $-0,01(0,01)$ & - & $-0,01(0,01)$ \\
\hline $\begin{array}{l}\text { EFI-L Soziale } \\
\text { Inklusion }\end{array}$ & - & $-0,00(0,01)$ & - & $-0,00(0,01)$ \\
\hline $\begin{array}{l}\text { EFI-L Persönli- } \\
\text { che Bereitschaft }\end{array}$ & - & $0,00(0,01)$ & - & $0,00(0,01)$ \\
\hline \multicolumn{5}{|c|}{ Modell Charakteristik } \\
\hline AIC & $-2516,35$ & $-2497,73$ & $-1047,79$ & $-1029,25$ \\
\hline $\mathrm{R}^{2} \mathrm{GLMM}(\mathrm{m})$ & 0,20 & 0,21 & 0,04 & 0,05 \\
\hline $\mathrm{R}^{2} \mathrm{GLMM}(\mathrm{c})$ & 0,41 & 0,41 & 0,11 & 0,11 \\
\hline $\begin{array}{l}\text { N (Kinder/ } \\
\text { Lehrkräfte) }\end{array}$ & $1494 / 84$ & $1494 / 84$ & $1490 / 84$ & $1490 / 84$ \\
\hline
\end{tabular}

SPF Sonderpädagogischer Förderbedarf, EFI-L Einstellungsfragebogen zu Inklusion für Lehrkräfte (Seifried und Heyl 2016)

$\mathrm{R}^{2}{ }_{\mathrm{GLMM}(\mathrm{m})}=$ marginales $\mathrm{R}^{2}, \mathrm{R}^{2} \mathrm{GLMM}(\mathrm{c})=$ konditionales $\mathrm{R}^{2}$ (nach Nakagawa und Schielzeth 2013). In Klammern sind die Standardfehler

*** $p \leq 0,001 ; * * p \leq 0,01 ; * p \leq 0,05$ 
Tab. 6 Mixed Effects Meta-Analysen der ERGMs für die Cross-Level-Interaktionen

\begin{tabular}{|c|c|c|c|c|}
\hline EFI-L & $\begin{array}{l}\text { Mixed Effects, SPF (einge- } \\
\text { hend) } \\
\text { Anzahl Netzwerke: } 73\end{array}$ & $\begin{array}{l}p \text { - } \\
\text { Wert }\end{array}$ & $\begin{array}{l}\text { Mixed Effects, SPF (ausge- } \\
\text { hend) } \\
\text { Anzahl Netzwerke: } 69\end{array}$ & $\begin{array}{l}p \text { - } \\
\text { Wert }\end{array}$ \\
\hline $\begin{array}{l}\text { Fachliche } \\
\text { Förderung }\end{array}$ & $-0,023(0,13)$ & 0,86 & $-0,111(0,12)$ & 0,36 \\
\hline $\begin{array}{l}\text { Soziale Inklusi- } \\
\text { on }\end{array}$ & $0,056(0,13)$ & 0,33 & $0,178(0,12)$ & 0,08 \\
\hline $\begin{array}{l}\text { Persönliche } \\
\text { Bereitschaft }\end{array}$ & $-0,117(0,12)$ & 0,67 & $-0,149(0,12)$ & 0,13 \\
\hline Gesamtscore & $-0,003(0,17)$ & 0,98 & $-0,078(0,16)$ & 0,63 \\
\hline
\end{tabular}

EFI-L Einstellungsfragebogen zu Inklusion für Lehrkräfte (Seifried und Heyl 2016)

Die Werte sind Koeffizienten, die immer im Vergleich zu einer Baseline (= Kinder ohne SPF) stehen. Ein negativer Wert bedeutet, dass Kinder mit SPF weniger Interaktionen im Vergleich zu den Kindern ohne SPF haben, wenn die Einstellung der Lehrkraft höher ist. In Klammern sind die Standardfehler

Die Meta-Analysen mit Mixed Effects Modellen zeigen, dass bei den eingehenden Interaktionen ein höherer Wert in der Einstellung der Lehrkraft mit einem tieferen Interaktionswert der Kinder mit SPF im Vergleich zum Interaktionswert der Kinder ohne SPF einhergeht (vgl. Tab. 6). Dies gilt für den EFI-L Gesamtscore, die Persönliche Bereitschaft zu inklusivem Unterricht und die Fachliche Förderung im inklusiven Unterricht, wobei keine Cross-Level-Interaktion signifikant ist ( $p$-Werte zwischen 0,67 und 0,98). Im Gegensatz dazu geht ein höherer Wert in der Einstellung zu Soziale Inklusion im Unterricht mit einem erhöhten eingehenden Interaktionswert der Kinder mit SPF im Vergleich zu Kindern ohne SPF einher. Aber auch dieser Effekt ist nicht signifikant $(p=0,33)$. Bei den ausgehenden Interaktionen bleiben die Richtungen der jeweiligen Effekte gleich. Sie fallen im Vergleich zu den eingehenden Interaktionen aber stärker aus. Allerdings ist auch bei den ausgehenden Interaktionen keine Cross-Level-Interaktion signifikant ( $p$-Werte zwischen 0,08 und 0,63). Das Ausmaß der sozialen Interaktionen der Kinder mit kognitiver Beeinträchtigung im Vergleich zu den Kindern ohne kognitive Beeinträchtigung wird nicht durch die Einstellung der Lehrkraft moderiert.

\section{Diskussion}

Zusammenfassend zeigen die Ergebnisse erstens, dass die Sportlehrkräfte eine positive Einstellung zu inklusivem Sportunterricht haben. Zweitens weisen Kinder mit SPF aufgrund einer kognitiven Beeinträchtigung signifikant weniger eingehende soziale Interaktionen auf als ihre Peers ohne SPF und sie nennen leicht mehr Interaktionen. Und drittens kann hinsichtlich der Bedeutung der Einstellung der Sportlehrkraft für die eingehenden und ausgehenden Interaktionen von Kindern im Sportunterricht weder ein Zusammenhang noch eine Cross-Level-Interaktion gefunden werden. Die Einstellungen der Sportlehrkräfte zu inklusivem Sportunterricht scheinen in keinem direkten Zusammenhang mit den sozialen Interaktionen der Schülerinnen und Schüler im inklusiven Sportunterricht zu stehen. 
Bezüglich der ersten Fragestellung weisen die Sportlehrkräfte in allen drei Subdimensionen des Einstellungsfragebogens signifikant höhere Werte auf als der theoretische Mittelwert. Des Weiteren liegen alle ausgewiesenen Werte deutlich höher als jene in einer Vergleichsstichprobe mit 186 Grundschullehrkräften aus Deutschland, in der dasselbe Instrument zur Messung der Einstellung verwendet wurde (Seifried 2015). Dabei muss aber das unterschiedliche Unterrichtssetting (Sport- vs. Klassenzimmerunterricht) berücksichtigt werden. Unser Befund geht einher mit den Resultaten von Reuker et al. (2016), die tendenziell von einer positiven Einstellung gegenüber inklusivem Sportunterricht berichten. Die zum Teil ambivalenten Befunde von Reuker et al. (2016) sind auch in der vorliegenden Studie ersichtlich: Es zeigt sich, dass nicht alle Subdimensionen gleich positiv eingeschätzt werden. Die affektiv-behaviorale Subdimension Persönliche Bereitschaft zu inklusivem Unterricht und die schülerbezogene, eher kognitive Subdimension Soziale Inklusion im Unterricht werden in der vorliegenden Studie am positivsten eingeschätzt. Lehrkräfte sind demnach bereit, inklusiv zu unterrichten und glauben an eine entsprechende Förderung der sozialen Partizipation, was zum Teil den Ergebnissen von Ewing et al. (2018) widerspricht. Das geringere Zutrauen in die Fachliche Förderung im inklusiven Unterricht könnte mit dem nach wie vor vorkommenden Sportarten- und Leistungsdenken im Sport einhergehen.

In der zweiten Fragestellung wurden die sozialen Interaktionen der Schülerinnen und Schüler anhand eines Peer-Ratings untersucht. Die vorliegenden Resultate im Sportunterricht gehen mit den Befunden aus der Grundschule einher, die eingeschränkte soziale Interaktionen von Kindern mit SPF in der inklusiven Bildung beschreiben (Garrote 2016; Henke et al. 2017a). Die vorliegenden Resultate zeigen, dass die Netzwerke in den Klassen von verschiedenen sozialen Prozessen geprägt sind - alle Prädiktoren bis auf den SPF sind auf einem Level von $p \leq 0,001$ signifikant (vgl. Tab. 4). Selbst wenn für endogene und anderweitig exogene Faktoren kontrolliert wird, weisen Kinder mit SPF signifikant weniger eingehende Interaktionen auf als Kinder ohne SPF. Bei den ausgehenden Interaktionen nennen Kinder mit SPF leicht mehr Interaktionen als Kinder ohne SPF, wie dies teilweise auch bei der Freundschaftsnennung bei Henke et al. (2017a) und Mamas et al. (2020) der Fall ist.

In der (sport-)pädagogischen Literatur wird häufig ein direkter Bezug von Lehrkrafteinstellungen zur tatsächlichen Unterrichtssituation angenommen. Die theoretische Grundlage für diese Annahme liefert das Kompetenzmodell von Baumert und Kunter (2013). Heyder et al. (2020) finden hingegen nur wenige empirische Studien, die diesen Zusammenhang überprüfen und kommen in ihrer eigenen Studie zum Schluss, dass nur spezifische Einstellungsfacetten der Lehrkräfte mit dem sozial-emotionalen Wohlbefinden der Schülerinnen und Schüler zusammenhängen. Bei der Multilevel-Regression bezüglich der dritten Fragestellung können wir keinen Nachweis für einen direkten Effekt der Lehrkrafteinstellung gegenüber inklusivem Sportunterricht und der soziometrisch erfassten sozialen Interaktionen von Kindern mit einer kognitiven Beeinträchtigung finden. Es scheint, dass Einstellungen von Lehrkräften keinen messbaren Einfluss auf die sozialen Interaktionen von Kindern mit kognitiver Beeinträchtigung haben. Andere Faktoren könnten diesbezüglich ein stärkeres Gewicht haben (z. B. Klassenklima, Klassennormen, Heterogenität). 
Weitere Gründe könnten zudem im spezifischen Setting des Sportunterrichts liegen: Im Vergleich zum Unterrichtsgeschehen im Klassenzimmer sind die Kinder im Sportunterricht mehr in Bewegung und haben dadurch per se mehr Interaktionsmöglichkeiten, was den potenziellen Einfluss der Lehrkraft in diesem Prozess verringern kann. Um mehr Einfluss zu nehmen, müssten die Sportlehrkräfte spezifisches, pädagogisches Wissen und Gestaltungsmöglichkeiten für den inklusiven Unterricht besitzen (Finkelstein et al. 2019). Hierzu müsste die Rolle der Lehrkraft und deren Einfluss auf die sozialen Interaktionen mittels tatsächlicher Unterrichtsgestaltung im Sportunterricht untersucht werden. Dies würde dann eher für eine indirekte Wirkung der Lehrkrafteinstellung auf die sozialen Prozesse auf Schülerinnen- und Schülerebene sprechen. Furrer et al. (2020) finden unter Berücksichtigung des SPF, des Geschlechts und psychomotorischer Fähigkeiten einen signifikanten Zusammenhang zwischen dem Gebrauch einer individuellen Bezugsnormorientierung bzw. dem Unterrichten von kooperativen Normen und Aspekten der sozialen Partizipation im inklusiven Sportunterricht. Schlussendlich könnte das Ausbleiben von Cross-Level-Interaktionen in der vorliegenden Studie daran liegen, dass der SPF ein robustes Attribut ist und ein möglicher Etikettierungseffekt von Kindern mit kognitiver Beeinträchtigung auch bei positiven Einstellungen der Lehrkräfte Bestand hat (vgl. Schwab 2019).

Nach unserem Wissen ist die vorliegende Studie die erste, die soziale Netzwerke im inklusiven Sportunterricht mittels ERGMs untersucht und diese in Zusammenhang mit der Einstellung der Lehrkraft zu inklusivem Sportunterricht stellt. Die verwendete statistische Auswertungsmethode eröffnet neue Erkenntnismöglichkeiten. So wurden in der bisherigen inklusiven Sportunterrichtsforschung netzwerkinhärente Strukturen (auch als Kontrollvariablen) vernachlässigt, obwohl diese - wie sich in unserer Studie zeigt - auf einer Metaebene eine signifikante Bedeutung auf die Strukturierung der sozialen Netzwerke haben.

\subsection{Limitierungen und Ausblick}

Limitierend gilt es festzuhalten, dass nur 84 von möglichen 109 Netzwerken berechnet werden konnten. Zusätzlich könnten noch weitere Kontrollvariablen fehlen, welche die geringeren sozialen Interaktionen von Kindern mit SPF erklären könnten (vgl. Henke et al. 2017b). Diesbezüglich ist weitere Forschung notwendig, die Variablen wie bspw. den sozioökonomischen Status oder konzeptionelle Fähigkeiten der Kinder in der Netzwerkanalyse mitberücksichtigen.

Des Weiteren müssen mögliche Selektionseffekte in der Stichprobengenerierung und soziale Erwünschtheit angenommen werden. Die entsprechend hohen Einstellungswerte könnten u. a. darauf zurückzuführen sein. Es wäre wünschenswert, wenn in zukünftigen Studien Zufallsstichproben gezogen werden können.

Schließlich wurden die auf einer 5-stufigen Likert-Skala erfassten sozialen Interaktionen für die ERGM Berechnungen dichotomisiert, wodurch viele relevante Informationen verloren gingen. Der Cut-off für die Dichotomisierung der Sprechkontakte wurde bei manchmal=0 gewählt. Weitere Analysen mit dem Cut-off bei manchmal=1 zeigen, dass sich nur der Effekt der ausgehenden Interaktionen von Kindern mit SPF ändert (Fixed Effects nicht mehr signifikant). Für zukünftige Stu- 
dien wäre es jedoch wünschenswert, wenn sogenannte ,valued networks“ in die Analyse miteinbezogen werden, mit welchen auf eine Dichotomisierung der Werte verzichtet werden kann (vgl. Krivitsky 2012). Eine weitere Ergänzung der quantitativen Instrumente der Soziometrie mit qualitativen Erhebungsmethoden könnte zusätzlich relevante Einblicke bei den Unterschieden in den sozialen Interaktionen ermöglichen.

\subsection{Konklusion}

Obwohl das innovative methodische Vorgehen in der vorliegenden Studie die angenommene Beziehung zwischen der Einstellung der Sportlehrkräfte und den sozialen Prozessen von Schülerinnen und Schülern im inklusiven Sportunterricht auf Grundschulstufe statistisch nicht belegt, ermöglicht sie detaillierte Einblicke in den inklusiven Sportunterricht: Die Befunde lassen auf eine Fachspezifik des Sportunterrichts schließen, da einerseits der Sportunterricht ein anderes Setting für Interaktionsmöglichkeiten darstellt und andererseits die Lehrkräfte in ihrer Rolle als Sportlehrkräfte eine positivere Einstellung gegenüber dem inklusiven Potenzial des Faches Bewegung und Sport zu haben scheinen als andere Lehrkräfte im Klassenzimmersetting (verglichen mit Resultaten von Seifried 2015). Da keine Cross-Level-Interaktion zwischen Einstellung der Sportlehrkraft und sozialen Interaktionen der Kinder mit SPF feststellbar ist, könnte dies dafürsprechen, dass die Einstellung der Lehrkraft im Sportunterricht wenig relevant für die sozialen Interaktionen ist - zumindest gibt es in der vorgelegten Studie keinen direkten Zusammenhang.

Ausgehend von den Befunden kann auch von einer gewissen Entlastung der Lehrkraft betreffend die Bedeutung ihrer Einstellung auf die sozialen Beziehungen der Schülerinnen und Schüler gesprochen werden. Dies ermöglicht es, den Fokus ausgeprägter auf die Veränderung der Sozialbeziehungen der Kinder im Sportunterricht zu lenken und dort den Hebel mit entsprechenden Unterrichtsmaßnahmen anzusetzen. So könnten die Sportlehrkräfte versuchen, bestehende Netzwerkstrukturen in der Sportklasse zu nutzen, um Kinder sozial besser zu integrieren. Eine Idee hierfür könnte es sein, Kinder mit SPF mit sehr aktiven Kindern in gemeinsamen Sportgruppen und -teams zusammenzubringen und voneinander lernen zu lassen (Klavina und Block 2008). Dadurch erhält das Kind mit SPF nicht nur mehr Kontakte, sondern die Wahrscheinlichkeit erhöht sich, dass das Kind eher in Sportgruppen eingebunden wird. Folglich könnte z. B. Kindern mit SPF der soziale Anschluss an ihre Mitschülerinnen und -schüler ermöglicht werden, indem entsprechende didaktische Settings gewählt werden, die soziale Interaktionen zwischen den Kindern fördern (z. B. Peer Tutoring). Um diese anspruchsvolle Aufgabe im Sportunterricht leisten zu können, scheinen entsprechende Weiterbildungsprogramme für Lehrkräfte bedeutsam.

Für die Weiterentwicklung inklusiver Lernsettings hinsichtlich ihrer sozialen Ziele braucht es noch weitere Studien, welche Einflussfaktoren für Aspekte der sozialen Partizipation untersuchen.

Danksagung Wir würdigen den wertvollen Beitrag der gesamten Forschungsgruppe im Projekt Soziale Partizipation im Sport (SoPariS): Thierry Schluchter, Pädagogische Hochschule Bern, Schweiz; Alexander Steiger, Universität Bern, Schweiz. 
Funding Open access funding provided by University of Teacher Education of Bern

Open Access Dieser Artikel wird unter der Creative Commons Namensnennung 4.0 International Lizenz veröffentlicht, welche die Nutzung, Vervielfältigung, Bearbeitung, Verbreitung und Wiedergabe in jeglichem Medium und Format erlaubt, sofern Sie den/die ursprünglichen Autor(en) und die Quelle ordnungsgemäß nennen, einen Link zur Creative Commons Lizenz beifügen und angeben, ob Änderungen vorgenommen wurden.

Die in diesem Artikel enthaltenen Bilder und sonstiges Drittmaterial unterliegen ebenfalls der genannten Creative Commons Lizenz, sofern sich aus der Abbildungslegende nichts anderes ergibt. Sofern das betreffende Material nicht unter der genannten Creative Commons Lizenz steht und die betreffende Handlung nicht nach gesetzlichen Vorschriften erlaubt ist, ist für die oben aufgeführten Weiterverwendungen des Materials die Einwilligung des jeweiligen Rechteinhabers einzuholen.

Weitere Details zur Lizenz entnehmen Sie bitte der Lizenzinformation auf http://creativecommons.org/ licenses/by/4.0/deed.de.

\section{Literatur}

Ajzen, I. (2005). Attitudes, personality and behavior (2. Aufl.). Mapping social psychology. Maidenhead: Open Univ. Press.

An, W. (2015). Multilevel meta network analysis with application to studying network dynamics of network interventions. Social Networks, 43, 48-56.

Baumeister, R.F., \& Leary, M.R. (1995). The need to belong: desire for interpersonal attachments as a fundamental human motivation. Psychological Bulletin, 117(3), 497-529.

Baumert, J., \& Kunter, M. (2013). The COACTIV model of teachers' professional competence. In M. Kunter, J. Baumert, W. Blum, U. Klusmann, S. Krauss \& M. Neubrand (Hrsg.), Cognitive activation in the mathematics classroom and professional competence of teachers: results from the COACTIV project. Mathematics teacher education, (Bd. 8, S. 25-48). Boston: Springer.

Ben-Yehuda, S., Leyser, Y., \& Last, U. (2010). Teacher educational beliefs and sociometric status of special educational needs (SEN) students in inclusive classrooms. International Journal of Inclusive Education, 14(1), 17-34.

Beni, S., Fletcher, T., \& Ní Chróinín, D. (2017). Meaningful experiences in physical education and youth sport: a review of the literature. Quest, 69(3), 291-312.

Boban, I., \& Hinz, A. (2003). Index für Inklusion - Lernen und Teilhabe in der Schule der Vielfalt entwickeln, Martin-Luther-Universität. https://www.eenet.org.uk/resources/docs/Index\%20German.pdf. Zugegriffen: 23. Okt. 2020

Borgatti, S.P., \& Quintane, E. (2018). Techniques: dichotomizing a network. Connections, 38(1), 1-11.

Butler, R. S., \& Hodge, S. R. (2004). Social inclusion of students with disabilities in middle school physical education classes ISSN. Research in Middle Level Education Online, 27(1), 1-10.

Casebolt, K. M., \& Hodge, S. R. (2010). High school physical education teachers' beliefs about teaching students with mild to severe disabilities. Physical Educator, 67(3), 140-155.

Cohen, J. (1988). Statistical power analysis for the behavioral sciences (2. Aufl.). Hillsdale: Erlbaum.

De Boer, A., Pijl, S. J., \& Minnaert, A. (2011). Regular primary schoolteachers' attitudes towards inclusive education: a review of the literature. International Journal of Inclusive Education, 15(3), 331-353.

De Boer, A., Pijl, S. J., Post, W., \& Minnaert, A. (2012). Peer acceptance and friendships of students with disabilities in general education: the role of child, peer, and classroom variables. Social Development, 35(3), 831-844.

Eckhart, M. (2012). Soziale Integrationsprozesse in Schulklassen: Methodische Annäherungen und Entwicklung eines Computerprogramms zur Analyse sozialer Prozesse in Schulklassen (Sozio). In A. Lanfranchi \& J. Steppacher (Hrsg.), Schulische Integration gelingt: Gute Praxis wahrnehmen, Neues entwickeln (S. 136-147). Bad Heilbrunn: Klinkhardt.

Ewing, D. L., Monsen, J. J., \& Kielblock, S. (2018). Teachers' attitudes towards inclusive education: a critical review of published questionnaires. Educational Psychology in Practice, 34(2), 150-165. 
Finkelstein, S., Sharma, U., \& Furlonger, B. (2019). The inclusive practices of classroom teachers: a scoping review and thematic analysis. International Journal of Inclusive Education. https://doi.org/10. 1080/13603116.2019.1572232.

Frostad, P., \& Pijl, S. J. (2007). Does being friendly help in making friends? The relation between the social position and social skills of pupils with special needs in mainstream education. European Journal of Special Needs Education, 22(1), 15-30.

Furrer, V., Valkanover, S., Eckhart, M., \& Nagel, S. (2020). The role of teaching strategies in social acceptance and interactions; considering students with intellectual disabilities in inclusive physical education. Frontiers in Education. https://doi.org/10.3389/feduc.2020.586960.

Garrote, A. (2016). Soziale Teilhabe von Kindern in inklusiven Klassen. Empirische Pädagogik, 30(1), 67-80.

Garrote, A., \& Moser Opitz, E. (2017). Soziale Interaktionen in Integrationsklassen fördern. Schweizerische Zeitschrift für Heilpädagogik, 23, 6-11.

Garrote, A., Sermier Dessemontet, R., \& Moser Opitz, E. (2017). Facilitating the social participation of pupils with special educational needs in mainstream schools: a review of school-based interventions. Educational Research Review, 20, 12-23.

Garrote, A., Felder, F., Krähenmann, H., Schnepel, S., Sermier Dessemontet, R., \& Moser Opitz, E. (2020). Social acceptance in inclusive classrooms: the role of teacher attitudes toward inclusion and classroom management. Frontiers in Education. https://doi.org/10.3389/feduc.2020.582873.

Goodwin, D.L. (2001). The meaning of help in PE: perceptions of students with physical disabilities. Adapted physical activity quarterly: APAQ, 18(3), 289-303.

Goodwin, D. L., \& Watkinson, E. J. (2000). Inclusive physical education from the perspective of students with physical disabilities. Adapted Physical Activity Quarterly, 17(2), 144-160.

Henke, T., Bogda, K., Lambrecht, J., Bosse, S., Koch, H., Maaz, K., \& Spörer, N. (2017a). Will you be my friend? A multilevel network analysis of friendships of students with and without special educational needs backgrounds in inclusive classrooms. Zeitschrift für Erziehungswissenschaft, 20(3), 449-474.

Henke, T., Bosse, S., Lambrecht, J., Jäntsch, C., Jaeuthe, J., \& Spörer, N. (2017b). Mittendrin oder nur dabei? Zum Zusammenhang zwischen sonderpädagogischem Förderbedarf und sozialer Partizipation von Grundschülerinnen und Grundschülern. Zeitschrift für Pädagogische Psychologie, 31(2), $111-123$.

Heyder, A., Südkamp, A., \& Steinmayr, R. (2020). How are teachers' attitudes toward inclusion related to the social-emotional school experiences of students with and without special educational needs? Learning and Individual Differences, 77, 101776.

Hunter, D. R., Handcock, M. S., Butts, C. T., Goodreau, S. M., \& Morris, M. (2008). ergm: a package to fit, simulate and diagnose exponential-family models for networks. Journal of statistical software, 24(3), nihpa54860.

Hutzler, Y., Meier, S., \& Reuker, S. (2017). Einstellung von Sportlehrkräften zu inklusivem Sportunterricht - mögliche Bezugspunkte (inter-)nationaler Forschung. Sonderpädagogische Förderung heute, 62(3), 244-254.

Hutzler, Y., Meier, S., Reuker, S., \& Zitomer, M. (2019). Attitudes and self-efficacy of physical education teachers toward inclusion of children with disabilities: a narrative review of international literature. Physical Education and Sport Pedagogy, 28(3), 1-18.

IBM Corp (2020). IBM SPSS statistics for Windows, version 27.0. Armonk: IBM Corp.

King, G. (2013). Perspectives on measuring participation: going forward. Child: care, health and development, 39(4), 466-469.

Klavina, A., \& Block, M.E. (2008). The effect of peer tutoring on interaction behaviours in inclusive physical education. Adapted Physical Activity Quarterly, 25(2), 132-158.

Koster, M., Nakken, H., Pijl, S. J., \& van Houten, E. (2009). Being part of the peer group: a literature study focusing on the social dimension of inclusion in education. International Journal of Inclusive Education, 13(2), 117-140.

Krivitsky, P. N. (2012). Exponential-family random graph models for valued networks. Electronic journal of statistics, 6, 1100-1128.

Lüke, T., \& Grosche, M. (2017). Konstruktion und Validierung der Professionsunabhängigen Einstellungsskala zum Inklusiven Schulsystem (PREIS). Empirische Sonderpädagogik, 10(1), 3-20.

Lusher, D., Robins, G., \& Koskinen, J. (Hrsg.). (2013). Exponential random graph models for social networks: theory, methods, and applications. Structural analysis in the social sciences, Bd. 35. Cambridge: Cambridge University Press.

Mamas, C., Bjorklund, P., Daly, A. J., \& Moukarzel, S. (2020). Friendship and support networks among students with disabilities in middle school. International Journal of Educational Research, 103, 101608. 
McPherson, M., Smith-Lovin, L., \& Cook, J. M. (2001). Birds of a feather: homophily in social networks. Annual Review of Sociology, 27(1), 415-444.

Monsen, J. J., Ewing, D. L., \& Kwoka, M. (2014). Teachers' attitudes towards inclusion, perceived adequacy of support and classroom learning environment. Learning Environments Research, 17(1), 113-126.

Nakagawa, S., \& Schielzeth, H. (2013). A general and simple method for obtaining R2 from generalized linear mixed-effects models. Methods in Ecology and Evolution, 4(2), 133-142.

Pinheiro, J., Bates, D., DebRoy, S., Sarkar, D., EISPACK authors, Heisterkamp, S., VanWilligen, B., \& R-core (2020). Linear and nonlinear mixed effects models: fit and compare Gaussian linear and nonlinear mixed-effects models. https://svn.r-project.org/R-packages/trunk/nlme/. Zugegriffen: 28. Okt. 2020.

Rekaa, H., Hanisch, H., \& Ytterhus, B. (2019). Inclusion in physical education: teacher attitudes and student experiences. A systematic review. International Journal of Disability, Development and Education, 66(1), 36-55.

Reuker, S., Rischke, A., Kämpfe, A., Schmitz, B., Teubert, H., Thissen, A., \& Wiethäuper, H. (2016). Inklusion im Sportunterricht. Sportwissenschaft, 46(2), 88-101.

Robins, G., \& Lusher, D. (2013). Illustrations: simulation, estimation and goodness of fit. In D. Lusher, G. Robins \& J. Koskinen (Hrsg.), Exponential random graph models for social networks: theory, methods, and applications. Structural analysis in the social sciences, (Bd. 35, S. 167-186). Cambridge: Cambridge University Press.

Schilling, F. (1976). Checklist motorischer Verhaltensweisen: Handanweisung für die Durchführung, Auswertung und Interpretation. Braunschweig: Georg Westermann.

Schwab, S. (2015). Einflussfaktoren auf die Einstellung von SchülerInnen gegenüber Peers mit unterschiedlichen Behinderungen. Zeitschrift für Entwicklungspsychologie und Pädagogische Psychologie, 47(4), 177-187.

Schwab, S. (2018). Attitudes towards inclusive schooling: a study on students', teachers' and parents' attitudes. Bd. 4. Münster: Waxmann.

Schwab, S. (2019). Friendship stability among students with and without special educational needs. Educational Studies, 45(3), 390-401.

Seifried, S., \& Heyl, V. (2016). Konstruktion und Validierung eines Einstellungsfragebogens zu Inklusion für Lehrkräfte (EFI-L). Empirische Sonderpädagogik, 1, 22-35.

Seifried, S. (2015). Einstellungen von Lehrkräften zu Inklusion und deren Bedeutung für den schulischen Implementierungsprozess - Entwicklung, Validierung und strukturgleichungsanalytische Modellierung der Skala EFI-L. Dissertation, Sonderpädagogik, Pädagogische Hochschule Heidelberg

Snijders, T.A.B., van de Bunt, G.G., \& Steglich, C.E.G. (2010). Introduction to stochastic actor-based models for network dynamics. Social Networks, 32(1), 44-60.

Valkanover, S. (2005). Intrigenspiel und Muskelkraft: Aspekte der Psychomotorik im Zusammenhang mit Mobbing im Kindergarten. Schulpädagogik - Fachdidaktik - Lehrerbildung, Bd. 11. Bern: Haupt.

Viechtbauer, W. (2010). Conducting meta-analyses in R with the metafor package. Journal of statistical software. https://doi.org/10.18637/jss.v036.i03.

Zander, L., \& Hannover, B. (2014). The self in educational contexts: how social networks shape self-related cognitions-and vice versa. Zeitschrift für Erziehungswissenschaft, 17(5), 225-240.

Vitus Furrer Wissenschaftlicher Assistent

Fabian Mumenthaler Wissenschaftlicher Assistent

Dr. Stefan Valkanover Dozent

Michael Eckhart Professor

Siegfried Nagel Professor 